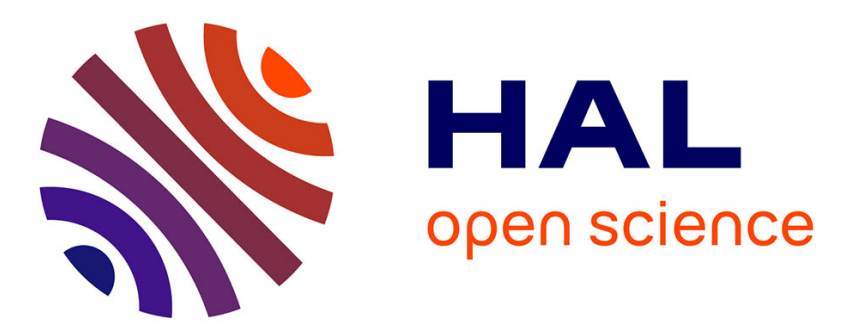

\title{
Frottement intérieur d'un magnésium de haute pureté déformé plastiquement
}

\author{
S.M. Seyedreihani, C. Esnouf, Gilbert Fantozzi, G. Revel
}

\section{To cite this version:}

S.M. Seyedreihani, C. Esnouf, Gilbert Fantozzi, G. Revel. Frottement intérieur d'un magnésium de haute pureté déformé plastiquement. Journal de Physique Lettres, 1978, 39 (22), pp.429-432. 10.1051/jphyslet:019780039022042900 . jpa-00231535

\section{HAL Id: jpa-00231535 https://hal.science/jpa-00231535}

Submitted on 1 Jan 1978

HAL is a multi-disciplinary open access archive for the deposit and dissemination of scientific research documents, whether they are published or not. The documents may come from teaching and research institutions in France or abroad, or from public or private research centers.
L'archive ouverte pluridisciplinaire HAL, est destinée au dépôt et à la diffusion de documents scientifiques de niveau recherche, publiés ou non, émanant des établissements d'enseignement et de recherche français ou étrangers, des laboratoires publics ou privés. 


\title{
FROTTEMENT INTÉRIEUR D'UN MAGNÉSIUM DE HAUTE PURETÉ DÉFORMÉ PLASTIQUEMENT
}

\author{
S. M. SEYED REIHANI, C. ESNOUF, G. FANTOZZI et G. REVEL (*)
}

Groupe d'Etudes de Métallurgie Physique et de Physique des Matériaux (**), Bât. 502 INSA, 69621 Villeurbanne Cedex, France

(*) Centre d'Etudes de Chimie Métallurgique, 15, rue Georges-Urbain, 94400 Vitry sur Seine, France

(Reçu le 3 juillet 1978, accepté le 27 septembre 1978)

\begin{abstract}
Résumé. - Nous avons étudié le frottement intérieur d'un magnésium de haute pureté (6N) après déformation plastique. Le spectre obtenu est très différent de celui observé jusqu'ici sur des matériaux moins purs. En effet, au lieu d'un très large maximum, nous constatons la présence : $a$ ) de deux pics situés vers $40 \mathrm{~K}$ et $80 \mathrm{~K}$ très stables lors des recuits, $b$ ) de deux pics situés vers $105 \mathrm{~K}$ et $220 \mathrm{~K}$ qui disparaissent lors de leur observation, $c$ ) d'un pic vers $15 \mathrm{~K}$ ou d'une diminution du frottement intérieur entre 10 et $30 \mathrm{~K}$ après recuit au-dessus de $300 \mathrm{~K}, d$ ) d'un pic stable vers $350 \mathrm{~K}$. Une tentative d'interprétation succincte de ces résultats est proposée.
\end{abstract}

\begin{abstract}
Internal friction of high purity $(6 \mathrm{~N})$ magnesium has been studied after plastic deformation. The internal friction spectrum is very different from that observed with the less pure magnesium. The broad peak observed for the less pure material was replaced by several peaks with the pure magnesium. These are : $a$ ) two peaks situated around 40 and $80 \mathrm{~K}$, stable during annealing, b) two peaks situated around $105 \mathrm{~K}$ and $220 \mathrm{~K}$, removed during temperature cycling, $c$ ) a peak at $15 \mathrm{~K}$ or an important decrease of internal friction between 10 and $30 \mathrm{~K}$ after annealing up to $300 \mathrm{~K}$, and $d$ ) a stable peak near to $350 \mathrm{~K}$. A brief interpretation of these observations is proposed.
\end{abstract}

1. Introduction. - Le frottement intérieur après déformation plastique a été abondamment étudié dans le cas des métaux c.f.c. $[1,2]$ : à basse température il apparaît d'une part la relaxation de Bordoni constituée des pics de Niblett et Wilks $\left(B_{1}\right)$ et de Bordoni $\left(B_{2}\right)$, d'autre part les pics d'interaction dislocationsdéfauts ponctuels ou de Hasiguti qui apparaissent à des températures supérieures à celles des pics de Bordoni. Les pics de Bordoni, contrairement aux pics de Hasiguti, sont stables au cours du recuit et de ce fait sont généralement attribués aux propriétés intrinsèques des dislocations.

Des études du même type ont été faites sur les métaux h.c., mais dans de nombreux cas, l'interprétation des phénomènes de relaxation observés semble moins évidente [2] et l'assimilation de ces phénomènes, soit à la relaxation de Bordoni, soit aux pics de Hasiguti, est très controversée.

En particulier Tsui et Sack [3] dans le domaine du kilohertz et Routbort [4] dans le domaine de l'hertz ont étudié le spectre de frottement intérieur du magnésium $99,996 \%$ mono- et polycristallin après laminage ou traction. Dans tous les cas, il apparaît un très large maximum de frottement intérieur qui s'étend

(**) ERA 463. entre 50 et $300 \mathrm{~K}$, dont l'amplitude dépend du taux de déformation, du traitement thermique et de l'orientation cristalline. De plus aucune variation de la température du maximum avec la fréquence n'a pu être mise en évidence, cette température restant voisine de $180 \mathrm{~K}$ pour un rapport de fréquence de $4 \times 10^{4}$. Ce résultat paraît relativement surprenant, même si l'on admet que le large maximum a une structure complexe due à une grande distribution des temps de relaxation des processus responsables.

Enfin Koda et al. [5] montrent que la relaxation obtenue au mégahertz est complexe et difficile à relier aux résultats obtenus à l'hertz ou au kilohertz.

Strunk et Frydman [6] soulignent que ces résultats pourraient être reliés au fait que les dislocations coin basales contiennent des crans dont les plans de glissement sont des plans pyramidaux ou prismatiques.

Dans ces conditions, il nous a paru intéressant de reprendre l'étude du frottement intérieur après déformation plastique en utilisant du magnésium de très haute pureté, afin de vérifier si les résultats obtenus jusqu'ici sont spécifiques ou non du magnésium.

2. Résultats expérimentaux. - Nous avons utilisé des échantillons polycristallins de magnésium de zone 
fondue $6 \mathrm{~N}$ [7] recuits sous argon $2 \mathrm{~h}$ à $300^{\circ} \mathrm{C}$ après filage (taille de grains $\simeq 1 \mathrm{~mm}$ ). Après les mesures de frottement intérieur, ces échantillons ont été ana- lysés par activation neutronique [8]. Le tableau indique les résultats obtenus pour les principales impuretés recherchées (poids analysé $10 \mathrm{mg}$ ).

\section{Analyse du magnésium utilisé}

[Analysis of the magnesium used.]

$\begin{array}{cccccccccccc}\begin{array}{c}\text { Principales } \\ \text { impuretés en }\end{array} & \mathrm{Cr} & \mathrm{Co} & \mathrm{Cu} & \mathrm{Fe} & \mathrm{Ga} & \mathrm{Hf} & \mathrm{La} & \mathrm{Mn} & \mathrm{Sb} & \text { W } & \mathrm{Zn} \\ \mu \mathrm{g} \cdot \mathrm{g}^{-1} & \leqslant 0,2 & 0,1 & 0,2 & \leqslant 0,07 & \leqslant 0,1 & \leqslant 0,002 & \leqslant 0,002 & 0,5 & \leqslant 0,004 & \leqslant 0,003 & 1\end{array}$

Les mesures de frottement intérieur ont été réalisées à l'aide d'un pendule de torsion inversé oscillant à une fréquence de l'ordre du hertz, asservi à amplitude constante $\left(\varepsilon_{\max } \simeq 2 \times 10^{-5}\right)$ et permettant l'enregistrement du frottement intérieur et de la période d'oscillation entre 10 et $500 \mathrm{~K}$ lors d'une montée linéaire en température de $50 \mathrm{~K} / \mathrm{h}$ [9].

La figure 1 présente le spectre de frottement intérieur obtenu directement après écrouissage in situ par torsion de $2,5 \%$ à $10 \mathrm{~K}$. Lors de la première montée en température (courbe 1), on observe essentiellement deux légères composantes vers 40 et $80 \mathrm{~K}$ et un pic important (décrément logarithmique de l'ordre de $5 \times 10^{-2}$ ) situé vers $105 \mathrm{~K}$. Après recuit à $150 \mathrm{~K}$ (courbe 2), il apparaît nettement un pic vers $45 \mathrm{~K}$, le pic à $105 \mathrm{~K}$ ayant disparu. De plus, lors de la deuxième montée linéaire (courbe 2) un pic situé vers $220 \mathrm{~K}$ est observé.

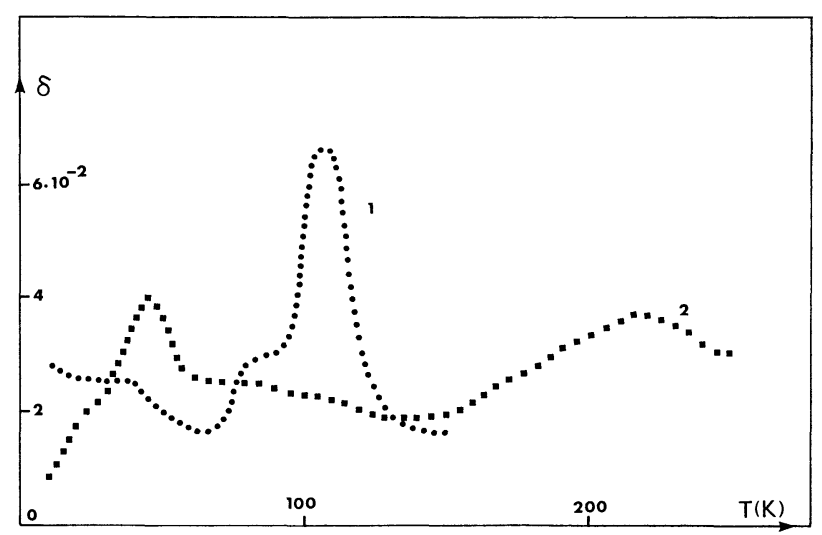

FIG. 1. - Spectre de frottement intérieur : 1) directement après déformation plastique par torsion de $2,5 \%$ à $10 \mathrm{~K}, 2$ ) après montée linéaire jusqu'à $150 \mathrm{~K}(f \simeq 1 \mathrm{~Hz})$.

[Internal friction spectrum as a function of temperature $: 1$ ) directly after plastic deformation of $2.5 \%$ at $10 \mathrm{~K}, 2)$ after heating up to $150 \mathrm{~K}(f \simeq 1 \mathrm{~Hz})$.]

Après recuit à $250 \mathrm{~K}$ (Fig. 2, courbe 1 ), le spectre de frottement intérieur est essentiellement constitué de deux pics situés respectivement vers $45 \mathrm{~K}$ et $75 \mathrm{~K}$. Après un maintien de 48 h à $295 \mathrm{~K}$ (Fig. 2, courbe 2), la hauteur du pic à $75 \mathrm{~K}$ est plus faible et le pic semble

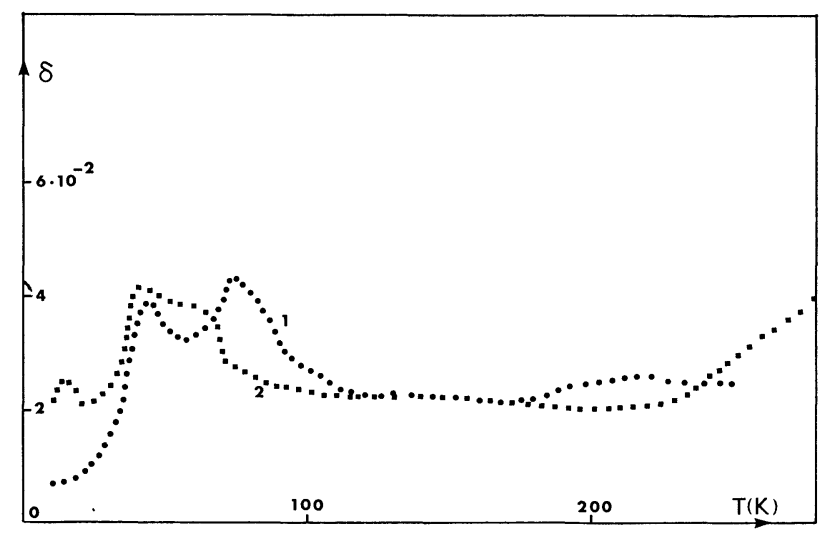

FIG. 2. - Spectre de frottement intérieur : 1) après montée linéaire à $250 \mathrm{~K}, 2$ ) après montée linéaire à $295 \mathrm{~K}$ et maintien de $48 \mathrm{~h}$.

[Internal friction spectrum as a function of temperature : 1) after heating up to $250 \mathrm{~K}, 2$ ) after annealing $48 \mathrm{~h}$ at $295 \mathrm{~K}$.]

se déplacer vers les basses températures. Il apparaît de plus, après ce maintien, un pic vers $15 \mathrm{~K}$.

Par recuit à plus haute température $(340,400,450$ et $500 \mathrm{~K}$ ) (Figs. 3 et 4), le pic à $75 \mathrm{~K}$ disparaît, le pic à $40 \mathrm{~K}$ étant plus stable (ce pic est toujours présent après recuit à $500 \mathrm{~K}$ ) (Fig. 4 , courbe 2 ). De plus, on

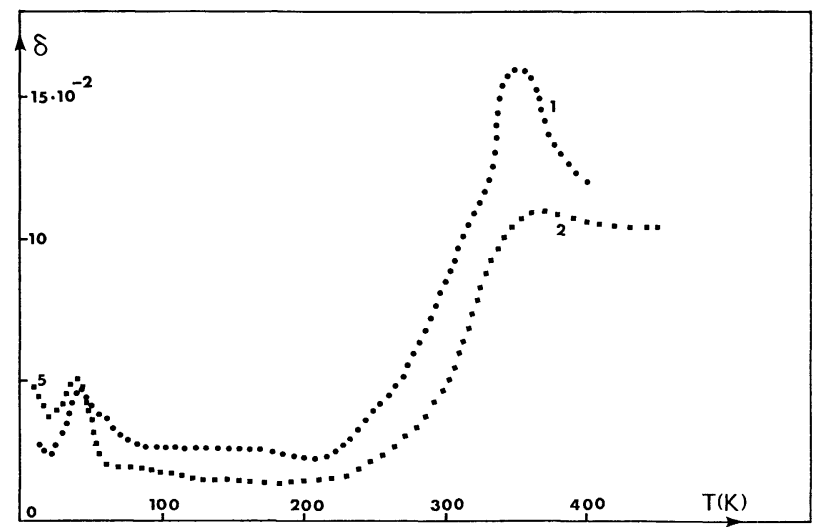

FIG. 3. - Spectre de frottement intérieur : 1) après montée linéaire à $340 \mathrm{~K}, 2$ ) après montée linéaire à $400 \mathrm{~K}$.

[Internal friction spectrum as a function of temperature : 1) after heating up to $340 \mathrm{~K}, 2$ ) after heating up to $400 \mathrm{~K}$.] 
observe, à $10 \mathrm{~K}$, une augmentation importante du frottement intérieur, qui est suivie d'une diminution rapide entre 10 et $30 \mathrm{~K}$.

En outre les figures 3 et 4 montrent la présence d'un pic stable situé vers $350 \mathrm{~K}$.

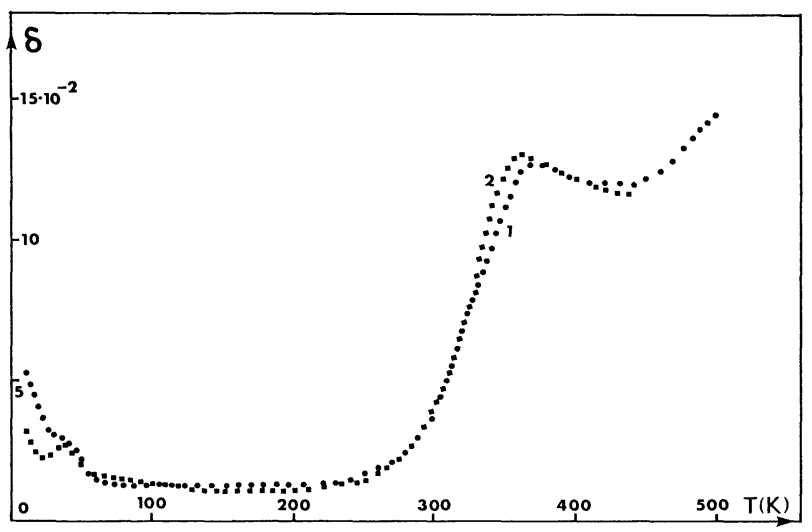

FIG. 4. - Frottement intérieur en fonction de la température : 1) après montée linéaire à $450 \mathrm{~K}, 2$ ) après montée linéaire à $500 \mathrm{~K}$.

[Internal friction spectrum as a function of temperature : 1) after heating up to $450 \mathrm{~K}, 2$ ) after heating up to $500 \mathrm{~K}$.]

3. Discussion. - Les résultats que nous avons obtenus sur un magnésium de très haute pureté sont sensiblement différents de ceux publiés jusqu'ici dans la littérature $[1,3,4,5]$. En effet, au lieu d'un maximum très large, nous constatons que le spectre de frottement intérieur est constitué de :

i) Deux pics situés vers 40 et $80 \mathrm{~K}$ environ, stables lors des recuits : ces pics apparaissent immédiatement après déformation plastique (Fig. 1, courbe 1) et ne disparaissent qu'après recuit à haute température (Fig. 4).

ii) Deux pics situés vers 105 et $220 \mathrm{~K}$ qui se distinguent des deux pics précédents par le fait qu'ils disparaissent au cours de leur observation.

iii) D'un pic vers $15 \mathrm{~K}$ ou d'une diminution importante du frottement intérieur entre 10 et $30 \mathrm{~K}$ après recuit au-dessus de $300 \mathrm{~K}$ (Figs. 2, 3 et 4).

iiii) D'un pic stable vers $350 \mathrm{~K}$.

Ce spectre de frottement intérieur est très proche de ceux observés sur l'ensemble des métaux c.f.c. [1, 2] et plus particulièrement de celui obtenu sur l'aluminium de haute pureté [10].

Par comparaison avec l'aluminium, nous pouvons donc proposer l'interprétation succincte suivante du spectre de frottement intérieur du magnésium après déformation plastique.

Parmi les pics observés, nous remarquons que deux d'entre eux ( $40 \mathrm{~K}$ et $80 \mathrm{~K}$ ) ont une bonne stabilité lors du recuit, un facteur d'élargissement de l'ordre de 3 à 4 et une température du maximum peu dépendante de l'état structural, caractéristiques correspondant à celles des pics de Bordoni dans les métaux c.f.c. Ainsi, les deux pics importants à 40 et $80 \mathrm{~K}$ peuvent être assimilés à la relaxation de Bordoni. La détermination que nous avons effectuée de l'énergie d'activation de chacun des pics par variation de fréquence (respectivement : $0,06<E<0,09 \mathrm{eV}$ pour le pic à $40 \mathrm{~K}$, et $0,10<E<0,15 \mathrm{eV}$ pour le pic à $80 \mathrm{~K})$ montre que les pics devraient être situés vers 80 et $150 \mathrm{~K}$ à $40 \mathrm{kHz}$. Bien qu'ils n'aient pas été observés jusqu'ici, il faut toutefois remarquer que les résultats de Tsui et Sack [3] font apparaître dans certains cas, deux légères composantes, situées respectivement vers 80 et $150 \mathrm{~K}$ qui correspondent aux deux pics observés.

Les deux pics à 105 et $220 \mathrm{~K}$ ont plutôt les caractéristiques des pics de Hasiguti [13] (dus à une interaction défauts ponctuels intrinsèques-dislocations) et semblent correspondre respectivement aux pics $\mathrm{P}_{\mathrm{A}}$ et $\mathrm{P}_{\mathrm{B}}$ de l'aluminium. Le défaut responsable du pic à $105 \mathrm{~K}$ est probablement un défaut de type interstitiel, ces défauts migrant en-dessous de $100 \mathrm{~K}$ environ [12]. Le pic à $220 \mathrm{~K}$ peut être associé soit à des amas d'interstitiels (comme pour le pic $P_{B}$ de l'aluminium [13]), soit à des défauts lacunaires, le domaine de température considéré correspondant à leur migration [12].

Les manifestations anélastiques observées à très basse température $(T<30 \mathrm{~K})$ ne peuvent être associées qu'au mouvement des décrochements géométriques, seuls susceptibles de se déplacer en-dessous des pics de Bordoni. Le fait d'observer un pic vers $15 \mathrm{~K}$ ou une diminution rapide du frottement intérieur entre 10 et $30 \mathrm{~K}$, nous incite à penser que le pic à $15 \mathrm{~K}$ se déplace vers les très basses températures $(<10 \mathrm{~K})$ au cours des recuits. Ce résultat avait déjà été observé dans un aluminium de haute pureté [14] et des expériences complémentaires paraissent nécessaires pour préciser ce point.

Enfin, le pic observé vers $350 \mathrm{~K}$ est comparable au pic mis en évidence dans un aluminium de haute pureté [15] et qui est dû soit au glissement des dislocations contrôlé par la montée des crans, soit au mouvement des dislocations contrôlé par le déplacement des nœuds par déviation thermiquement activée [16].

En conclusion, les premiers résultats obtenus sur un magnésium de haute pureté montrent que le spectre de frottement intérieur après déformation plastique est très voisin de celui généralement observé dans les métaux c.f.c., contrairement à ce qui avait été signalé jusqu'ici sur du magnésium moins pur.

Remerciements. - Nous tenons à remercier le Dr. C. Boujour de l'Ecole Polytechnique Fédérale de Lausanne pour les intéressantes et fructueuses discussions que nous avons eu avec lui. 


\section{Bibliographie}

[1] Nowick, A. S. and Berry, B. S., Anelastic relaxation in crystalline solids (Academic Press, New York) 1972

[2] Fantozzi, G., Benoit, W., Esnouf, C. et Perez, J., Ann. Phys., à paraître.

[3] Tsui, R. T. C. et Sack, H. S., Acta Met. 15 (1967) 1715.

[4] Routbort, Thesis, Cornell University, New York (1965).

[5] Koda, S., Kamigaki, K. et Kayano, H., J. Phys. Soc. Japan 18 suppl. I (1963) 195.

[6] Strunk, H. et Frydman, R., Mat. Sci. Eng. 18 (1975) 143.

[7] Revel, G., Postal, J. L. et Rouchaud, J. C., C. R. Hebd. Séan. Acad. Sci. Paris 281C (1975) 1065.

[8] Revel, G., Fedoroff, M., Nucl. Instrum. Methods 143 (1977) 277.
[9] Esnouf, C., Fantozzi, G. and Gobin, P. F., Mem. Sci. Rev. Met. 71 (1974) 637.

[10] EsNouf, C., Thèse, Université Claude-Bernard et INSA (1978).

[11] Seeger, A., Philos. Mag. 1 (1956) 651.

[12] Delaplace, J., Hillairet, J., Nicoud, J. C., Phys. Status Solidi 30 (1968) 119.

[13] Perez, J., Peguin, P., Fantozzi, G. et Gobin, P., Ann. Phys. 5 (1970) 503.

[14] Esnouf, C., Fantozzi, G., Pernoux, E. and Gobin, P. F., Nuovo Cimento 33B (1976) 160.

[15] Esnouf, C., Gabbay, M. et Fantozzi, G., J. Physique Lett. 38 (1977) L-401.

[16] Friedel, J., J. Physique Lett. 39 (1978) L-61. 